\title{
Insuficiência Renal Aguda após Intervenção Coronária Percutânea Primária no Infarto Agudo do Miocárdio: Preditores e Evolução Clínica a Longo Prazo
}

\author{
Raphael Lanza e Passos ${ }^{1}$, Dimytri Alexandre A. Siqueira ${ }^{1}$, José Fabio Almiro Silva ${ }^{1}$, \\ Francisco Carleial Feijó de Sá ${ }^{1}$, José de Ribamar Costa Junior ${ }^{1}$, Fausto Feres' ${ }^{1}$, Alexandre Abizaid ${ }^{1}$, \\ Áurea J. Chaves ${ }^{1}$, Rodolfo Staico ${ }^{1}$, Luiz Alberto Mattos ${ }^{1}$, Amanda G. M. R. Sousa ${ }^{1}$, J. Eduardo Sousa ${ }^{1}$
}

\section{RESUMO}

Fundamentos: A ocorrência de insuficiência renal aguda (IRA) está associada a pior evolução clínica após infarto agudo do miocárdio (IAM), sendo sua ocorrência de natureza multifatorial. Os preditores e o prognóstico da IRA após intervenção coronária percutânea (ICP) primária ainda não estão bem estabelecidos. Objetivo: Identificar os preditores de IRA em pacientes submetidos a ICP primária e determinar a evolução clínica a longo prazo. Método: Estudo retrospectivo, no qual foram incluídos pacientes com quadro de IAM com supra-ST submetidos a ICP primária, no período de janeiro de 2002 a janeiro de 2007. Os pacientes foram classificados quanto à presença de IRA (aumento da creatinina $\geq 0,5 \mathrm{mg} / \mathrm{dl}$ ou $>25 \%$ em relação aos valores basais) durante evolução intra-hospitalar. Os pacientes foram acompanhados clinicamente por meio de revisão de prontuário e contato telefônico para avaliação de eventos maiores. Resultados: Foram incluídos 150 pacientes com média de idade de 60 anos, sendo $73 \%$ do sexo masculino. Fatores considerados indicadores de risco de IRA, como diabetes melito (34\%), Killip $>1(27 \%)$, volume de contraste e fármacos, também foram avaliados. IRA ocorreu em $15,3 \%$ dos pacientes. Após análises univariada e multivariada: creatinina $\geq 1,5 \mathrm{mg} / \mathrm{dl}$ (odds ratio [OR]: 3,633; intervalo de confiança de 95\% [IC 95\%]: $1,047-12,613 ; p=0,042)$, aumento da idade (OR: 1,066; IC 95\%: 1,002-1,090; $p=0,044)$, apresentação ou ocorrência de Killip > 1 (OR: 3,190; IC 95\%: 1,025-9,933; $p=0,045)$ e necessidade de ventilação mecânica (OR: 6,364; IC 95\%: 2,142-18,910; $p=0,001)$ foram considerados preditores independentes de IRA. Após um ano de evolução, os eventos óbito $(p=0,001)$ e reinfarto $(p=0,048)$ apresentaram diferença significativa entre os grupos com e sem IRA; tal diferença não ocorreu para revascularização $(p=0,305)$, diálise $(p=0,281)$ e eventos combinados $(p=0,060)$. Essas diferenças permaneceram na evolução de seis anos.

\section{SUMMARY}

Acute Renal Failure after Primary Percutaneous

Coronary Intervention in Acute Myocardial Infarction: Predictors and Long-Term Clinical Evolution

Background: The occurrence of acute renal failure (ARF) is associated with worse clinical outcomes after acute myocardial infarction (AMI), and its nature is multifactorial. The predictors and prognosis for ARF after primary percutaneous coronary intervention $(\mathrm{PCl})$ are still not well established. Objective: To identify the predictors of ARF in patients submitted to primary $\mathrm{PCl}$ and to determine long term clinical evolution. Methods: This is a retrospective study which includes patients with ST-segment elevation myocardial infarction (STEMI) submitted to primary $\mathrm{PCl}$ from January, 2002 to January, 2007. Patients were classified with respect to ARF - an increase in creatinine $\geq 0.5 \mathrm{mg} / \mathrm{dl}$ or $>25 \%$ from baseline - during hospital stay. Follow-up data were obtained through medical records or by telephone contact to assess major events. Results: A total of 150 patients were enrolled. Mean age was 60 years old and $73 \%$ were men. Factors considered risk indicators for ARF such as diabetes mellitus (34\%), Killip $>1(27 \%)$, contrast volume and use of potential nephrotoxic drugs were also evaluated. ARF occurred in $15.3 \%$ of patients. After multivariate analyses: creatinine $\geq 1.5 \mathrm{mg} / \mathrm{dl}$ (OR 3.633 95\% Cl 1.047-12.613 p = 0.042), older age (OR 1.066 95\% Cl 1.002-1.090, $\mathrm{p}=0.044)$, Killip $>1$ (OR $3.19095 \% \mathrm{Cl} 1.025-9.933, \mathrm{p}=0.045)$ and the need for mechanical ventilation (OR $6.36495 \%$ Cl 2.142-18.910, $p=0.001$ ) were considered independent predictors for ARF. After a year of evolution, death $(p=0.001)$ and reinfarction $(p=0.048)$ had shown a significant difference between patients with and without ARF; differences were not found for revascularization $(p=0.305)$, dialysis $(p=0.281)$ or

Instituto Dante Pazzanese de Cardiologia - São Paulo, SP.

Correspondência: Raphael Lanza e Passos. Rua José Antonio CoeIho, 661/24 - Vila Mariana - São Paulo, SP - CEP 04011-061 E-mail: raphaellanza@hotmail.com

Recebido em: 4/8/2008 • Aceito em: 29/10/2008 
Conclusão: Os principais preditores de IRA após ICP primária relacionam-se com idade, presença de creatinina $\geq 1,5 \mathrm{mg} / \mathrm{dl}$ e má evolução clínica intra-hospitalar. IRA após ICP primária constitui preditor de eventos maiores a curto e longo prazos.

DESCRITORES: Angioplastia transluminal percutânea coronária. Infarto do miocárdio. Insuficiência renal aguda. Meios de contraste/efeitos adversos. Rim/efeitos de drogas.

A intervenção coronária percutânea (ICP) primária é o tratamento de escolha no infarto agudo do miocárdio com supradesnivelamento do segmento ST (IAMCSST), pois se relaciona com maior patência do vaso, preservação da função ventricular esquerda e melhor sobrevida quando comparado à terapia trombolítica ${ }^{1}$. Dentre as potenciais complicações ocorridas na evolução intra-hospitalar de pacientes submetidos a angioplastia primária, a insuficiência renal aguda (IRA) tem sido destacada como importante fator prognóstico, associando-se a hospitalização mais prolongada e a maior morbidade e mortalidade ${ }^{2-4}$.

Mehran et al. ${ }^{5}$ avaliaram os vários fatores de risco para nefropatia induzida por contraste, determinando um escore de estratificação de risco para o desenvolvimento de IRA após ICP. Excluíram pacientes tratados com ICP por infarto agudo do miocárdio e aqueles com choque. Os preditores de nefropatia induzida por contraste foram presença de hipotensão, uso de balão intra-aórtico, insuficiência cardíaca, creatinina basal $>1,5 \mathrm{mg} / \mathrm{dl}$, idade inferior a 75 anos, diabetes melito, anemia e volume de contraste utilizado.

O desenvolvimento de IRA após procedimentos de urgência (ICP primária) é de natureza multifatorial e com incidência considerável. Dentre as particularidades que contribuem para a ocorrência de IRA nessas situações, destacam-se a presença de hipotensão ou choque, a utilização de maior volume de contraste e a impossibilidade de se realizar estratégias terapêuticas de prevenção da nefropatia induzida pelo contraste, em especial a pré-hidratação com solução salina.

Os preditores e o prognóstico a longo prazo da IRA após ICP primária ainda não estão bem estabelecidos. O objetivo do presente estudo foi determinar a incidência e os preditores de ocorrência de IRA após a angioplastia primária, bem como a evolução clínica a longo prazo dos pacientes que apresentaram essa complicação durante o tratamento inicial do IAMCSST. combined events $(p=0.060)$. The differences found for death and reinfarction remained significant after six years of evolution. Conclusion: The main predictors of ARF after primary $\mathrm{PCl}$ were age, baseline creatinine $\geq 1.5 \mathrm{mg} / \mathrm{dl}$ and poor clinical evolution during hospital stay. ARF after primary $\mathrm{PCl}$ is a predictor of major events in the short and long-term follow-up.

DESCRIPTORS: Angioplasty, transluminal, percutaneous coronary. Myocardial infarction. Renal insufficiency, acute. Contrast media/adverse effects. Kidney/drug effects.

\section{MÉTODO \\ Delineamento}

Estudo observacional, realizado em único centro (Instituto Dante Pazzanese de Cardiologia - São Paulo, SP), com o objetivo primário de determinar a incidência, os preditores de IRA após ICP primária e a evolução clínica dos pacientes conforme a ocorrência de eventos cardíacos adversos maiores (óbito, reinfarto, nova revascularização e necessidade de diálise).

\section{Seleção de pacientes}

No período compreendido entre janeiro de 2002 e janeiro de 2007, selecionamos todos os pacientes consecutivamente submetidos a ICP primária para o tratamento de IAMCSST por meio do levantamento dos pacientes em nosso banco de dados, posteriormente realizando avaliação dos prontuários (prescrições, exames laboratoriais, dados clínicos, evolução clínica, dados do procedimento, etc.) e, por fim, contato telefônico para avaliação de eventos clínicos. Como critérios de inclusão, os pacientes deveriam apresentar-se com até doze horas do início dos sintomas, com eletrocardiograma revelando supradesnivelamento $>1 \mathrm{~mm}$ em duas ou mais derivações contíguas ou bloqueio de ramo esquerdo; em casos de choque cardiogênico decorrentes de IAMCSST, a janela de apresentação era de até dezoito horas.

$\mathrm{Na}$ admissão hospitalar, amostra de sangue para dosagem de creatinina sérica basal deveria ter sido coletada, com novas dosagens seriadas de creatinina sérica ao longo dos primeiros três dias após a realização de ICP primária. Pacientes nos quais não fossem realizadas ao menos duas dosagens (incluindo-se a basal) de creatinina sérica foram excluídos (6\%), bem como pacientes submetidos a angioplastia facilitada ou a cirurgia de revascularização na fase intra-hospitalar.

\section{Definições}

A ocorrência de IRA foi definida pelo aumento da creatinina $\geq 0,5 \mathrm{mg} / \mathrm{dl}$ ou aumento $\geq 25 \%$ em relação aos valores basais, durante a evolução intra-hospitalar. 
Lanza e Passos R, et al. Insuficiência Renal Aguda após Intervenção Coronária Percutânea Primária no Infarto Agudo do Miocárdio: Preditores e Evolução Clínica a Longo Prazo. Rev Bras Cardiol Invas. 2008;16(4):422-428.

Óbito de origem cardíaca foi definido como morte em razão de evento cardiovascular (infarto do miocárdio, baixo débito cardíaco, arritmias fatais).

Infarto agudo do miocárdio foi definido como novas ondas $\mathrm{Q}$ patológicas em duas ou mais derivações contíguas, elevação da creatina quinase (CK) ou sua isoenzima MB (CK-MB) três vezes maior que o valor superior da normalidade durante a hospitalização ou duas vezes maior que o limite superior da normalidade após a alta hospitalar.

O diagnóstico de reinfarto requeria reelevação da CK ou da CK-MB 50\% maior que o menor valor previamente encontrado, associado à recorrência de sintomas ou a novas alterações eletrocardiográficas. Definiu-se como revascularização do vaso-alvo a ocorrência de novo procedimento de revascularização percutânea ou cirúrgica de qualquer segmento do vaso previamente tratado.

\section{Intervenção coronária percutânea e farmacologia adjunta}

Os procedimentos de revascularização foram realizados conforme diretrizes nacionais e internacionais de ICP, visando à obtenção de implante ótimo de stents à análise angiográfica. Todos os pacientes foram pré-tratados com ácido acetilsalicílico (AAS) na dose de $300 \mathrm{mg}$ associado a clopidogrel na dose de 300-600 mg e a heparina endovenosa na dose de 70-100 UI/kg. Inibidores da glicoproteína IIb/IIla foram utilizados conforme a avaliação do cardiologista intervencionista. Foi utilizado contraste iônico de baixa osmolaridade (Hexabrix ${ }^{\oplus}$, ioxaglato) em todos os pacientes. Os stents utilizados foram, em sua maioria, não-farmacológicos.

Medicamentos com ação anti-plaquetária - AAS $200 \mathrm{mg} / \mathrm{dia}$ e clopidogrel $75 \mathrm{mg} / \mathrm{dia}$ ou ticlopidina $500 \mathrm{mg} / \mathrm{dia}$ - foram prescritos durante a internação e mantidos por trinta dias a doze meses após o procedimento, na dependência do stent implantado (farmacológico vs. não-farmacológico).

\section{Acompanhamento clínico}

O acompanhamento clínico foi realizado no Ambulatório de Angioplastia Coronária da própria instituição ou por meio de contato telefônico, visando à análise da ocorrência de eventos clínicos adversos.

\section{Análise estatística}

As variáveis contínuas foram expressas em média e desvio padrão e as variáveis categóricas foram expressas em números absolutos e porcentagem (\%). Na análise univariada, as diferenças entre as variáveis contínuas foram determinadas pelo teste $t$ de Student. A análise de diferenças entre as variáveis categóricas foi realizada com os testes do qui-quadrado de Pearson, exato de Fisher e Mann-Whitney. Para a análise multi- variada foi utilizada regressão logística de Cox. As curvas de sobrevida livre de eventos foram obtidas pelo método de Kaplan-Meier e as diferenças foram identificadas pelo teste de log-rank. Valores de $p<0,05$ foram considerados estatisticamente significantes. A análise estatística foi realizada com o uso do Statistical Package for Social Sciences versão 11.0.

\section{RESULTADOS}

\section{Incidência e características clínicas}

De um total de 150 indivíduos selecionados, IRA após a angioplastia primária ocorreu em 23 (15,3\%) pacientes. A média de idade foi de $60 \pm 11,81$ anos, sendo $73 \%$ dos pacientes do sexo masculino. Cerca de $34 \%$ dos pacientes eram diabéticos e $12,6 \%$ apresentavam creatinina $\geq 1,5 \mathrm{mg} / \mathrm{dl}$ previamente à ICP. As características clínicas dos pacientes que desenvolveram IRA e daqueles que não apresentaram essa complicação encontram-se na Tabela 1. Comparativamente, os pacientes que evoluíram com IRA eram mais idosos e apresentavam, mais freqüentemente, creatinina basal elevada $(\geq 1,5 \mathrm{mg} / \mathrm{dl})$, classe funcional mais avançada (Killip > 2), além de necessidade de suporte hemodinâmico e ventilatório (uso de aminas vasoativas e de ventilação mecânica).

Os dados relacionados ao procedimento estão dispostos na Tabela 2. Não foram observadas diferenças significativas entre os grupos no que se refere ao vaso tratado e ao diâmetro e à extensão dos stents utilizados. Inibidores de glicoproteína IIb/IIla foram requeridos em 48,6\% das intervenções. Em relação ao volume de contraste utilizado, não foi observada diferença estatística entre os grupos (média de $145 \pm$ $80,41 \mathrm{ml}$ para o grupo com IRA e de $157 \pm 73,3 \mathrm{ml}$ para o grupo sem IRA; $p=0,339$ ), e sua distribuição encontra-se representada na Figura 1. Analisamos, ainda, a utilização de fármacos com potencial de nefrotoxicidade durante a ICP e na evolução intra-hospitalar. Nenhum dos fármacos, conforme demonstrado na Figura 2, esteve correlacionado à ocorrência de IRA nessa população.

\section{Preditores independentes de insuficiência renal aguda}

Após análise multivariada, idade (odds ratio [OR]: 1,066; intervalo de confiança de 95\% [IC 95\%]:1,002-1,090; $p=0,044)$, presença de creatinina basal $\geq 1,5 \mathrm{mg} / \mathrm{dl}$ (OR: 3,633; IC 95\%: 1,047-12,613; $p=0,042$ ), apresentação ou ocorrência de Killip > 1 (OR: 3,190; IC 95\%: $1,025-9,933 ; p=0,045)$ e necessidade de ventilação mecânica (OR: 6,364; IC 95\%: 2,142-18,910; $p=0,001$ ) foram identificados preditores independentes de IRA após a ICP.

\section{Insuficiência renal aguda e evolução clínica}

Os pacientes com IRA apresentaram pior evolução clínica quando comparados àqueles que não de- 
TABELA 1

Características clínicas

\begin{tabular}{lccc}
\hline Base de dados & \multicolumn{3}{c}{ Insuficiência renal aguda } \\
\cline { 2 - 4 } & Sim $(\mathbf{n}=\mathbf{2 3})$ & Não $(\mathbf{n}=\mathbf{1 2 7})$ & Valor de $\boldsymbol{p}$ \\
\hline Idade (média), anos & $66 \pm 11,94$ & $60 \pm 11,81$ & 0,037 \\
Sexo masculino,\% & 66,7 & 73,3 & 0,598 \\
Hipertensão,\% $\%$ & 66,4 & 0,120 \\
Diabetes melito,\% & 85 & 39,7 & 0,440 \\
Dislipidemia,\% & 30 & 54,3 & 0,093 \\
Tabagismo,\% & 75 & 36,2 & 0,917 \\
ATC pré, $\%$ & 35 & 14,7 & 0,960 \\
RM pré,\% & 15 & 8,6 & 0,370 \\
IAM pré,\% & 15 & 23,3 & 0,574 \\
Peso (média), kg & 30 & $72 \pm 14,22$ & 0,698 \\
Altura (média), metros & $69,9 \pm 9,02$ & $1,64 \pm 0,07$ & 0,996 \\
Creatinina $\geq 1,5$ mg/dl & $1,65 \pm 0,05$ & 11,4 & 0,048 \\
Troponina (média),, gg/l & 28,6 & $55,47 \pm 35,79$ & 0,685 \\
CK-MB (média), IU/l & $60,29 \pm 34,84$ & $130 \pm 93,04$ & 0,164 \\
Killip $\geq 2, \%$ & $91,5 \pm 66,13$ & 25,9 & 0,018 \\
Amina vasoativa,\% & 40 & 17,2 & 0,033 \\
Ventilação mecânica,\% & 40 & 9,5 & 0,002 \\
\hline
\end{tabular}

$\mathrm{p}<0,05$ = significativo.

ATC = angioplastia transluminal coronária; IAM = infarto agudo do miocárdio; $\mathrm{n}$ = número de pacientes; RM = revasculariação do miocárdio.

TABELA 2

Características do procedimento

\begin{tabular}{lccc}
\hline Dados do procedimento & \multicolumn{3}{c}{ Insuficiência renal aguda } \\
\cline { 2 - 4 } & Sim $(\mathbf{n}=\mathbf{2 3})$ & Não $(\mathbf{n}=\mathbf{1 2 7})$ & Valor de $\boldsymbol{p}$ \\
\hline Volume de contraste (média), $\mathrm{ml}$ & $145 \pm 80,41$ & $157 \pm 73,30$ & 0,341 \\
Diâmetro do stent (média), mm & $3,19 \pm 0,25$ & $3,10 \pm 0,36$ & 0,733 \\
Comprimento do stent (média), mm & $20,22 \pm 4,37$ & $19,91 \pm 4,59$ & 0,392 \\
Vaso (\%) & & & 0,292 \\
TCE & 0 & 2,6 & \\
DA & 45 & 44 & \\
CX & 5 & 13,8 & \\
CD & 50 & 16,2 & \\
MG & 0 & 0,7 & \\
VP & 0 & 0,9 & \\
\hline
\end{tabular}

$p>0,05=$ significativo.

$\mathrm{CD}=$ artéria coronária direita; $\mathrm{CX}=$ artéria circunflexa; $\mathrm{DA}=$ artéria descendente anterior; $\mathrm{MG}=$ artéria marginal da $\mathrm{CX} ; \mathrm{n}=\mathrm{número}$ de pacientes; TCE = tronco de artéria coronária esquerda; VP = artéria ventricular posterior.

senvolveram essa complicação em seguimentos a médio e longo prazos. No seguimento clínico de até um ano, representado na Figura 3, óbito e reinfarto foram mais freqüentes nos pacientes que evoluíram com IRA durante a internação $(30,4 \%$ vs. 5,5\%, p =0,001 e $10 \%$ vs. $1,8 \%, p=0,048$, respectivamente). Após acom- panhamento de cerca de seis anos, as diferenças nas curvas de sobrevida livre de óbito e de reinfarto em pacientes com e sem IRA se mantiveram, com maior ocorrência desses eventos em pacientes com IRA (Figuras 4 e 5). Nova revascularização ocorreu nos pacientes que evoluíram com e sem IRA, respectivamen- 


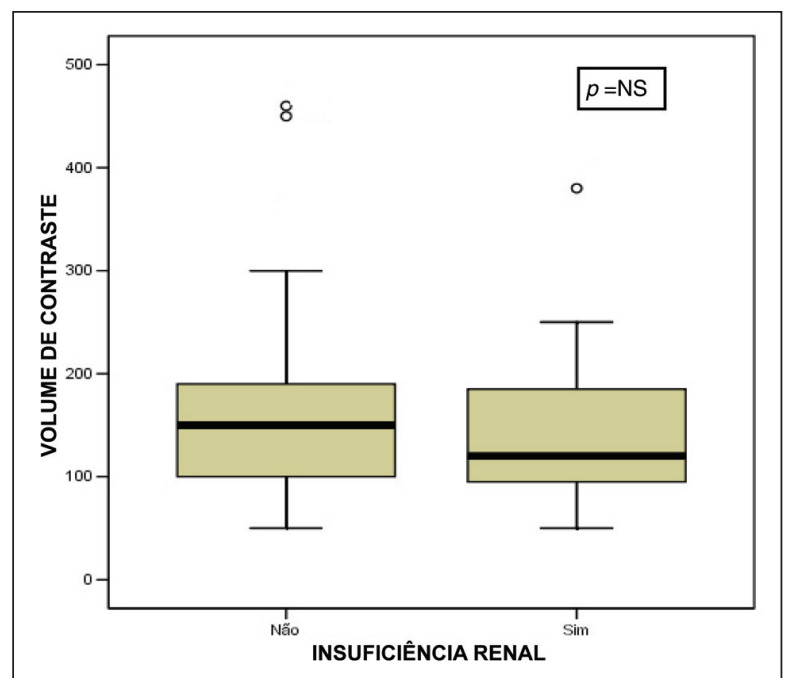

Figura 1 - O volume de contraste utilizado por paciente foi semelhante entre os grupos com média menor que $200 \mathrm{ml}$. NS = nãosignificativo; $p=0,341=$ NS.

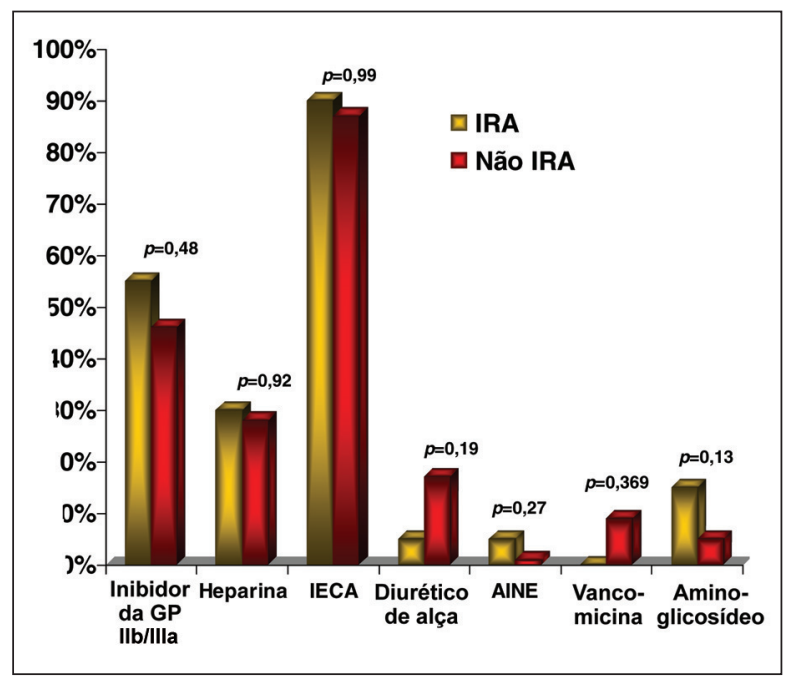

Figura 2 - Utilização de fármacos com potencial de nefrotoxicidade durante a ICP e na evolução intra-hospitalar. AINE = antiinflamatório não-esteróide; GP = glicoproteína; IECA = inibidor da enzima conversora da angiotensina; $\mathrm{p}<0,05=$ significativo.

te, em $5 \%$ vs. $15,9 \%(p=0,305)$, e necessidade de diálise em $5 \%$ vs. $0,9 \%(p=0,281)$. Os eventos combinados (óbito, reinfarto, revascularização, diálise) ocorreram em $45 \%$ dos pacientes que evoluíram com IRA intra-hospitalar e em $24,8 \%$ dos pacientes que não evoluíram com IRA ( $p=0,060)$.

\section{DISCUSSÃo}

Neste estudo, a IRA foi uma complicação freqüente após a ICP primária no IAMCSST, ocorrendo em $15,3 \%$ dos pacientes, mesmo naqueles com função renal prévia normal $(10,6 \%)$. Resultados semelhantes foram encontrados por Marenzi et al. ${ }^{6}$ (19\% de IRA) e

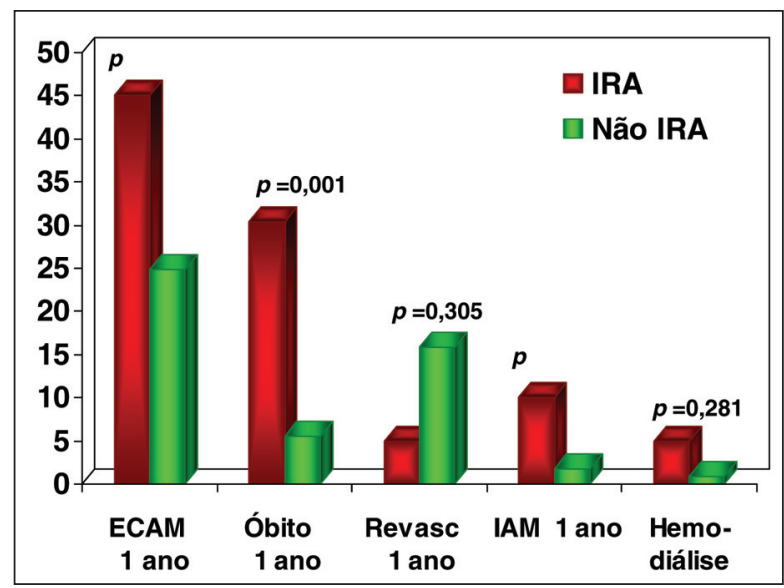

Figura 3 - Eventos maiores em até um ano. ECAM = eventos cardíacos adversos maiores (óbito, revascularização, reinfarto, diálise); IAM = reinfarto; IRA = insuficiência renal aguda; $\operatorname{Revasc}=$ revascularização; $p<0,05=$ significativo.

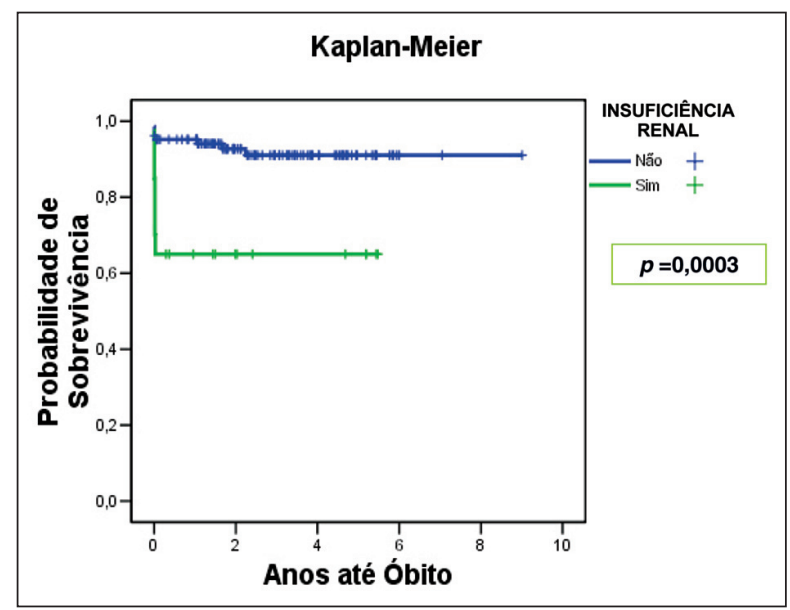

Figura 4 - Curva de sobrevida livre de óbito. $p<0,05=$ significativo.

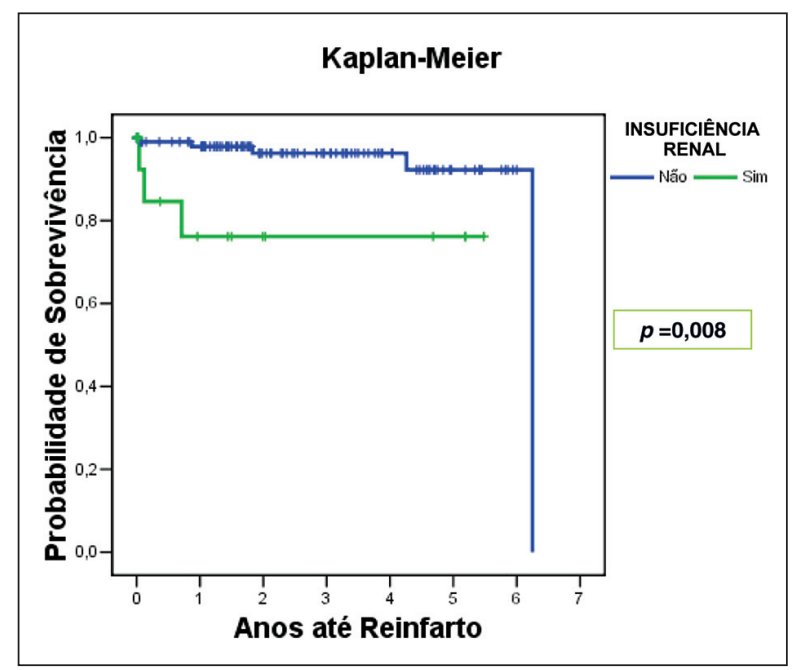

Figura 5 - Curva de sobrevida livre de reinfarto. $p<0,05=$ significativo. 
Lanza e Passos R, et al. Insuficiência Renal Aguda após Intervenção Coronária Percutânea Primária no Infarto Agudo do Miocárdio: Preditores e Evolução Clínica a Longo Prazo. Rev Bras Cardiol Invas. 2008;16(4):422-428.

Sadeghi et al. $^{2}$ (18\% de IRA) após ICP primária. A IRA esteve associada a pior evolução clínica intra-hospitalar (maior freqüência de Killip > 1, necessidade de uso de amina vasoativa e ventilação mecânica), com maior mortalidade e morbidade a longo prazo.

A nefropatia induzida por contraste se caracteriza por uma alteração renal ocorrida após administração de contraste na ausência de outras causas de falência renal $^{4}$. No paciente submetido a ICP primária, a deterioração da função renal ocorre de maneira multifatorial. Marenzi et al. ${ }^{6}$ avaliaram a relação entre volume de contraste e IRA na ICP primária e apontaram que $300 \mathrm{ml}$ seria uma quantidade de contraste preditor de IRA. Em nosso estudo, não encontramos relação entre contraste e IRA, o que, provavelmente, se deve a alguns fatores: utilizamos média de contraste bem inferior (cerca de $150 \mathrm{ml}$ por paciente - diagnóstico e intervenção) àquela apontada como preditor pela literatura na ICP primária; a amostra pode ter sido pequena para avaliar tal relação; outros fatores apresentaram maior relevância na indução de IRA, amenizando a influência do fator contraste.

Cada vez mais a ICP primária se afirma como terapia de escolha no tratamento do IAMCSST, por restaurar de forma mais rápida e eficiente o fluxo Coronariano e, conseqüentemente, por preservar a função ventricular, diminuindo a morbidade e a mortalidade nesses pacientes ${ }^{1,7}$. A instabilidade clínica no IAMCSST ocorre por causas variadas, tais como disfunção ventricular, síndrome da resposta inflamatória sistêmica e disfunção valvar, entre outras. No presente estudo, avaliamos a instabilidade clínica por meio da apresentação clínica segundo a classificação de Killip, do uso de drogas vasoativas e da necessidade de ventilação mecânica. Essas variáveis se mostraram preditoras de IRA e, conseqüentemente, indicadoras de má evolução clínica a curto e longo prazos. Zijlstra et al. ${ }^{7}$ e Keeley et al. ${ }^{1}$, por outro lado, avaliaram a função ventricular após ICP primária, demonstrando que os pacientes que evoluíam com disfunção ventricular apresentavam maior risco de IRA e pior evolução clínica.

Estudos prévios têm demonstrado que a creatinina basal e o estágio de evolução da doença renal são fatores de risco independentes para óbito após ICP primáriaa ${ }^{2,6,8}$. Mehran et al. ${ }^{5}$ demonstraram como preditor independente de IRA, em estudo que culminou no escore de risco de nefropatia induzida por contraste, a creatinina basal $>1,5 \mathrm{mg} / \mathrm{dl}$. Quando avaliamos a creatinina basal em nossa população, observamos que a incidência de IRA era mais freqüente no grupo com creatinina $\geq 1,5 \mathrm{mg} / \mathrm{dl}$ em comparação com o grupo com creatinina normal $(28,6 \%$ vs. $11,4 \%)$.

O aumento do risco de desenvolver IRA também tem sido reportado em pacientes com insuficiência renal crônica com síndrome coronariana sem supradesnivelamento do segmento ST submetidos a ICP preco-
$\mathrm{Ce}^{3,9}$. Nesse grupo de pacientes, a profilaxia de IRA pode ser realizada e normalmente é possível obter a estabilidade clínica dos pacientes previamente à intervenção, diferentemente dos pacientes com IAMCSST submetidos a ICP primária, nos quais não é possível a realização de hidratação prévia e a instabilidade hemodinâmica é mais freqüente.

Os estudos que avaliaram disfunção renal no infarto agudo do miocárdio são unânimes em afirmar que os pacientes com IRA apresentam evolução desfavorável, tanto imediata como a longo prazo ${ }^{10,11}$. Marenzi et al. ${ }^{6}$ referem, em amostra de 208 pacientes, mortalidade intra-hospitalar de $31 \%$ para os pacientes com IRA e de $0,6 \%$ para aqueles sem IRA $(p<0,001)$. Sadeghi et al. ${ }^{2}$, ao compararem grupos de pacientes com e sem IRA, encontraram, em seguimento de trinta dias, mortalidade de $7,5 \%$ vs. $0,8 \%(p<0,0001)$ e em seguimento de um ano, mortalidade de $12,7 \%$ vs. $2,4 \%$ $(p<0,0001)$, respectivamente. O presente trabalho confirmou esses dados, demonstrando que os pacientes submetidos a ICP primária que desenvolveram IRA $(15,3 \%)$ apresentaram curvas de sobrevida livre de óbito e revascularização desfavoráveis em relação àqueles pacientes que não desenvolveram IRA, tanto em acompanhamento de um ano como de cerca de seis anos (Figuras 3 a 7 ).

Marenzi et al. ${ }^{12}$ avaliaram a eficácia da $\mathrm{N}$-acetilcisteína na ICP primária em doses habituais associadas a hidratação pós-procedimento $(600$ mg antes e duas vezes por dia durante 48 horas pós-procedimento), em doses elevadas associadas a hidratação pós-procedimento (1.200 mg antes e duas vezes por dia durante 48 horas pós-procedimento), e somente com hidratação pós-procedimento. Os autores obtiveram resultados significativos no que se refere à diminuição de IRA e de eventos maiores (óbito, necessidade de diálise e ventilação mecânica) favoráveis à $\mathrm{N}$-acetilcisteína, sendo

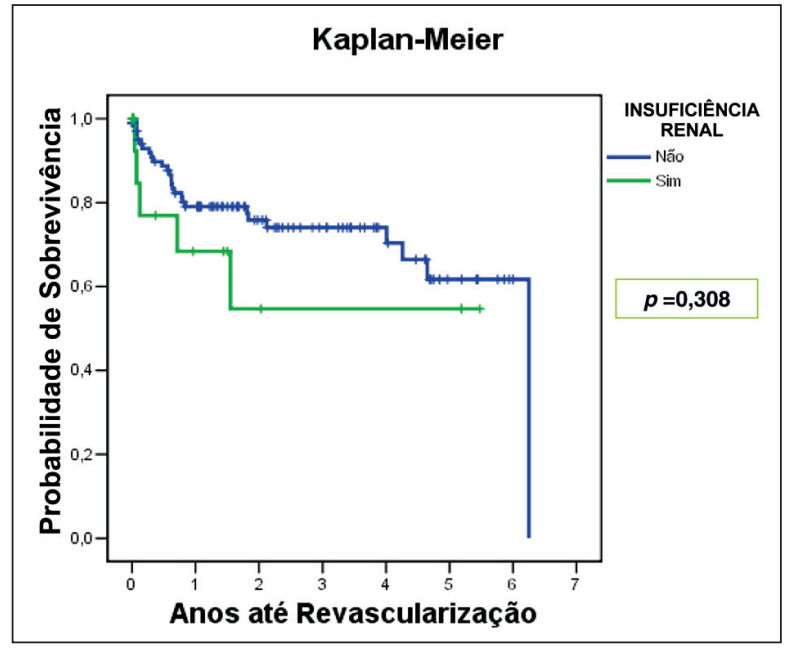

Figura 6 - Curva de sobrevida livre de revascularização. $p<0,05=$ significativo. 


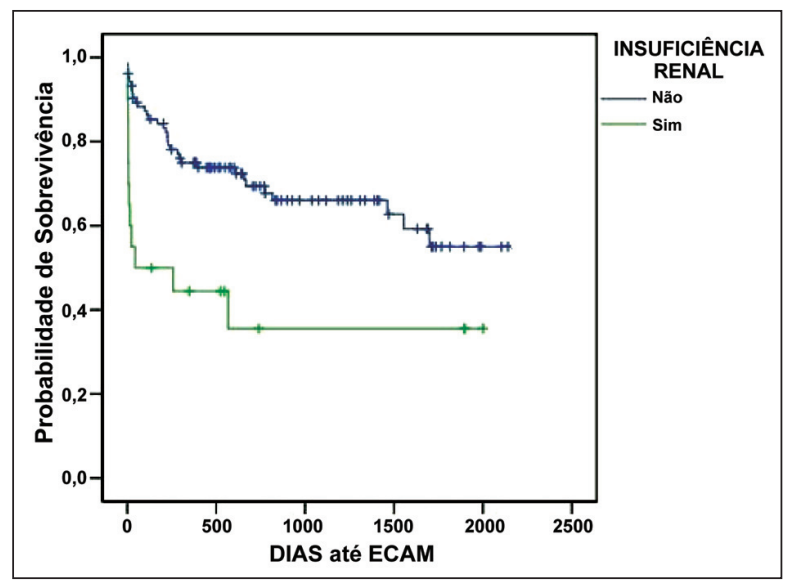

Figura 7 - Curva de sobrevivência livre de óbito, reinfarto, revascularização e diálise. $\mathrm{ECAM}=$ eventos cardíacos adversos maiores. $p=0,06=$ não-significativo.

a dose de $1.200 \mathrm{mg}$ melhor que a dose de $600 \mathrm{mg}$. São poucos os trabalhos que avaliaram a prevenção de IRA na ICP primária, uma vez que os pacientes com IRA apresentam pior evolução clínica a curto e longo prazos. Novas estratégias de prevenção de IRA devem ser desenvolvidas e testadas mesmo em pacientes com função renal normal. Deve-se, também, realizar o tratamento precoce otimizado e agressivo dos pacientes com IAMCSST, procurando-se manter a estabilidade clínica, principalmente no caso de pacientes idosos, grupo que apresenta alto risco para desenvolver IRA.

\section{Limitações}

O presente estudo é retrospectivo e, portanto, possui todas as limitações inerentes ao modelo. A definição de doença renal crônica não foi dada pela taxa de filtração glomerular estimada, mas pela creatinina basal $(\geq 1,5 \mathrm{mg} / \mathrm{dl})$, o que poderia subestimar sua real prevalência nessa população. Além disso, não foi possível avaliar o estado de hidratação pré- e pós-procedimento, fator que certamente influenciou a evolução da função renal. Por fim, o número de pacientes do estudo pode não ter sido suficiente para detectar o valor prognóstico de algumas variáveis, como volume de contraste ou medicações adjuntas.

\section{CONCLUSÃO}

Os principais preditores de IRA após ICP primária relacionam-se com a idade, a presença de creatinina $\geq 1,5 \mathrm{mg} / \mathrm{dl}$ e a má evolução clínica intra-hospitalar. IRA após ICP primária constitui preditor de eventos maiores a curto e longo prazos.

\section{REFERÊNCIAS BIBLIOGRÁFICAS}

1. Keeley EC, Boura JA, Grines CL. Primary angioplasty versus intravenous thrombolytic therapy for acute myocardial infarction: a quantitative review of 23 randomised trials. Lancet. 2003;361(9351):13-20.

2. Sadeghi HM, Stone GW, Grines CL, Mehran R, Dixon SR, Lansky AJ, et al. Impact of renal insufficiency in patients undergoing primary angioplasty for acute myocardial infarction. Circulation. 2003;108(22):2769-75.

3. Wison S, Foo K, Cunningham J, Cooper J, Deaner A, Knight $C$, et al. Renal function and risk stratification in acute coronary syndromes. Am J Cardiol. 2003;91(9):1051-4.

4. Toprak O, Cirit M. Risk factors for contrast-induced nephropathy. Kidney Blood Press Res. 2006;29(2):84-93.

5. Mehran R, Aymong ED, Nikolsky E, Lasic Z, lakovou I, Fahy $M$, et al. A simple risk score for prediction of contrastinduced nephropathy after percutaneous coronary intervention: development and initial validation. J Am Coll Cardiol. 2004;44(7):1393-9.

6. Marenzi G, Lauri G, Assanelli E, Campodonico J, De Metrio M, Marana I, et al. Contrast-induced nephropathy in patients undergoing primary angioplasty for acute myocardial infarction. J Am Coll Cardiol. 2004;44(9):1780-5.

7. Zijlstra F, Hoorntje JC, de Boer MJ, Reiffers S, Miedema K, Ottervanger JP, et al. Long-term benefit of primary angioplasty as compared with thrombolytic therapy for acute myocardial infarction. N Engl J Med. 1999;341(19):1413-9.

8. Hannan EL, Racz MJ, Arani DT, Ryan TJ, Walford G, Mecallister BD. Short- and long-term mortality for patients undergoing primary angioplasty for acute myocardial infarction. J Am Coll Cardiol. 2000;36(4):1194-201.

9. Freeman RV, Mehta RH, Al Badr W, Cooper JV, Kline-Rogers E, Eagle KA. Influence of concurrent renal dysfunction on outcomes of patients with acute coronary syndromes and implications of the use of glycoprotein Ilb/Illa inhibitors. J Am Coll Cardiol. 2003;41(5):718-24.

10. Al Suwaidi J, Reddan DN, Williams K, Pieper KS, Harrington RA, Califf RM, et al. Prognostic implications of abnormalities in renal function in patients with acute coronary syndromes. Circulation. 2002;106(8):974-80.

11. Yamaguchi J, Kasanuki H, Ishii $Y$, Yagi $M$, Nagashima $M$, Fujii $S$, et al. Serum creatinine on admission predicts longterm mortality in acute myocardial infarction patients undergoing successful primary angioplasty: data from the Heart Institute of Japan Acute Myocardial Infarction (HIJAMI) Registry. Circ J. 2007;71(9):1354-9.

12. Marenzi G, Assanelli E, Marana I, Lauri G, Campodonico J, Grazi $M$, et al. N-acetylcysteine and contrast-induced nephropathy in primary angioplasty. N Engl J Med. 2006;354(26): 2773-82. 